\title{
Fracturas apendiculares en caninos politraumatizados, complicaciones asociadas y tratamientos actuales
}

\section{Appendicular fractures in polytraumatized canines, associated complications and current treatments}

DOI: $10.46932 / \mathrm{sfjdv2n2-096}$

Received in: March 1st, 2021

Accepted in: May 30th, 2021

\author{
Esmeralda Dioselina Pimbosa O. \\ Magister en Clínica y Cirugía Canina. \\ Universidad Técnica de Machala, Facultad de Ciencias Agropecuarias. Escuela de Medicina \\ Veterinaria. Machala, Ecuador. \\ E-mail: dpimbosa@utmachala.edu.ec
}

\begin{abstract}
RESUMEN
La presencia de animales politraumatizados es frecuente en la práctica diaria del médico veterinario, entre las patologías óseas más frecuentes son las fracturas del esqueleto apendicular, estas pueden venir acompañadas de lesiones torácicas, abdominales, craneales y vertebrales, por lo cual, se ha planteado como objetivo de la investigación: analizar las diferentes complicaciones asociadas a las fracturas del esqueleto apendicular en caninos y los tratamientos que existen en la actualidad, para esto se recurrió a la investigación bibliográfica de diferentes bases de datos, las publicaciones científicas revisadas corresponden a los últimos cinco años; entre los temas tratados en la investigación se detallan las complicaciones asociadas a las fracturas apendiculares en caninos, elementos de osteosíntesis utilizados frecuentemente en el tratamiento de fracturas apendiculares, tratamiento cerrado u ortopédico de fracturas apendiculares, entre otros, los cuales fueron descritos en la literatura, las principales complicaciones asociadas con las fracturas apendiculares en caninos, las lesiones de otros tejidos aparte del hueso, y el abordaje radiológico mediante el uso de los diferentes elementos de fijación utilizados para tratar al paciente, de lo cual se concluye que en la actualidad los métodos a los que se recurre con mayor frecuencia para el tratamiento de fracturas apendiculares son los métodos de fijación interna, principalmente los tornillos, placas y clavos intramedulares, de estos, debido a sus ventajas, entre estas: menor agresión de partes blandas, menor tiempo quirúrgico, mínimo abordaje quirúrgico y su extracción es fácil.
\end{abstract}

Palabras clave: patología, fractura, lesiones, canino, tratamiento, tejidos

\begin{abstract}
The presence of polytraumatized animals is frequent in the daily practice of the veterinarian, among the most frequent bone pathologies are the fractures of the appendicular skeleton, these can be accompanied by thoracic, abdominal, cranial and vertebral injuries, for which, it has been considered as Objective of the research: to analyze the different complications associated with fractures of the appendicular skeleton in canines and the treatments that currently exist, for this we resorted to bibliographic research of different databases, the reviewed scientific publications correspond to the last five years; Among the topics covered in the research are the complications associated with appendicular fractures in canines, osteosynthesis elements frequently used in the treatment of appendicular fractures, closed or orthopedic treatment of appendicular fractures, among others, which were described in the literature. the main complications associated with appendicular fractures in canines, injuries to tissues other than bone, and the radiological approach through the use of the different fixation elements used to treat the patient, from which it is concluded that currently the methods Those most frequently used for the treatment of appendicular
\end{abstract}


fractures are internal fixation methods, mainly screws, plates and intramedullary nails, of these, due to their advantages, among these: less aggression of soft tissues, less surgical time , minimal surgical approach and its extraction is easy.

Key words: pathology, fracture, injuries, canine, treatment, tissues

\section{INTRODUCCIÓN}

El término politraumatismo no dispone de una definición que sea aceptada universalmente, sin embargo, esta palabra se utiliza para describir a aquellos pacientes con traumas y lesiones graves que afectan a varias regiones del cuerpo; generalmente se espera que un paciente en estas condiciones tenga mayor riesgo de mortalidad en relación con la sumatoria de la mortalidad que se espera de las lesiones individuales (Hsieh et al., 2018).

Una validación piloto recomienda explicar el politraumatismo como un paciente que supera la puntuación de 2 en la escala abreviada de lesiones, en por lo menos dos regiones del cuerpo (Butcher et al., 2014). El caso de pacientes con politraumatismo es menos frecuente en humanos (von Ritgen et al., 2017). En la práctica diaria del médico veterinario es frecuente la presencia de animales atropellados por peatones y caídas (De Morais et al., 2015).

Las patologías óseas más frecuentes son las fracturas del esqueleto apendicular (FEA), estas pueden venir acompañadas de lesiones torácicas, abdominales, craneales y vertebrales (Sánchez et al., 2017). Las FEA se pueden tratar mediante métodos cerrados, métodos abiertos, y métodos externos con reducción; en la actualidad la idea es trabajar con la reducción más fisiológica posible (García \& Ortega, 2005).

Este trabajo de investigación tiene como objetivo analizar las diferentes complicaciones asociadas a las fracturas del esqueleto apendicular en caninos y los tratamientos que existen en la actualidad, para esto se recurrió a la investigación bibliográfica de diferentes bases de datos, las publicaciones científicas revisadas corresponden a los últimos cinco años.

\section{MATERIALES Y MÉTODOS}

De acuerdo con el objetivo propuesto, las publicaciones científicas revisadas fueron valoradas de acuerdo con la metodología PRISMA. Primero se revisó las publicaciones científicas relacionadas con el tema investigado, estas se analizaron a profundidad para la obtención de inferencias adecuadas para la problemática. Se siguió el siguiente protocolo de investigación: 


\section{PROTOCOLO DE INVESTIGACIÓN}

Figura 1. Protocolo de investigación

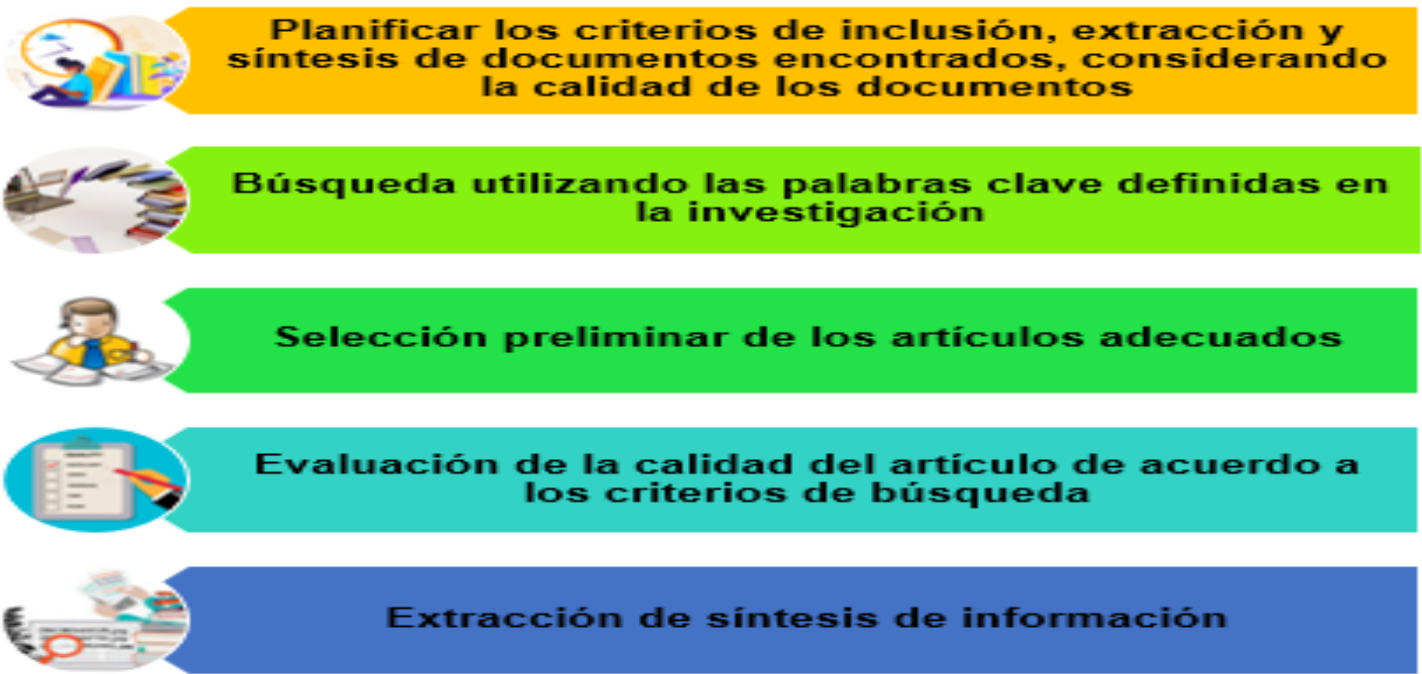

\section{COMPLICACIONES ASOCIADAS A LAS FRACTURAS APENDICULARES EN CANINOS}

\subsection{SHOCK HIPOVOLÉMICO}

El shock hipovolémico está asociado con varios procesos de enfermedad, entre estos: hemorragias, vómitos y fracturas apendiculares; como consecuencia, se presenta una reducción del suministro de oxígeno tisular asociado con la hipovolemia, si esto no se reconoce a tiempo puede desarrollarse una disfunción multiorgánica y finalmente la muerte (Llewellyn et al., 2020).

Las causas que ocasionan el shock hipovolémico en el animal pueden ser cardiogénicas, sépticas o hipovolémicas, todas tienen como resultado el inadecuado suministro de oxígeno a los tejidos; en el caso de animales politraumatizados la causa más frecuente es la presencia de hemorragias, principalmente en el abdomen, pecho o en el lugar donde se ha presentado alguna fractura, esto puede ser en los huesos largos o la pelvis (Rozanski \& Rondeau, 2002).

Todo paciente con politraumatismos debe ser valuado con la finalidad de buscar evidencias de shock hipovolémico, este se manifiesta por medio de la palidez de las membranas mucosas, tiempo de llenado capilar prolongado, taquicardia, extremidades frías y mala calidad del pulso periférico, estos síntomas son comunes en pacientes que han cursado cuadros hemorrágicos significativos, sean estas externas o intracavitarias (Balakrishnan, 2020).

El shock hipovolémico compensatorio y descompensatorio temprano está asociado con taquicardia refleja en los perros y en algunas ocasiones también en los gatos, esta se puede corregir mediante el uso de la fluidoterapia en perros y gatos hipovolémicos (Byers, 2017). 


\section{EMBOLIA GRASA}

En las fracturas diafisarias del fémur en ocasiones es posible observar la presencia de la embolia grasa (Agbelele et al., 2020); consiste en la presencia de grasa macroscópica que se instala dentro de la vasculatura del individuo, su ocurrencia es alta cuando el paciente ha presentado traumatismos, a pesar de esto, es poco frecuente observar grasa venosa en el diagnóstico por imágenes del sitio donde se produjo el trauma (Burr et al., 2020).

Los hallazgos de embolia grasa son de difícil detección cuando la presión intratoráxica es alta, o está bastante lesionada la caja torácica (Hitosugi et al., 2019). Los signos en el paciente que ha sufrido de fracturas de huesos largos se presentan de 24 a 48 horas después del accidente, el cuadro clínico es característico (Almeida et al., 2020).

Probablemente los síntomas clínicos del síndrome de embolia grasa sean una combinación entre oclusión vascular mecánica, la réplica inflamatoria del cuerpo al trauma y la grasa embolizada (Rothberg \& Makarewich, 2018).

\section{SÍNDROME COMPARTIMENTAL AGUDO}

Se trata de una emergencia ortopédica, la morbilidad y mortalidad pueden ser potencialmente devastadoras (Scollan et al., 2020). Se presenta cuando se incrementa la presión en un espacio cerrado, hasta el punto de reducir el flujo sanguíneo y modifica la perfusión tisular, como consecuencia es frecuente observar isquemia, y en ocasiones posibles necrosis en este espacio (Mahmoud \& Mahmoud, 2018).

El síndrome compartimental agudo suele acompañar a las fracturas, el 50\% de estos casos representan las fracturas abiertas (Sohn et al., 2019). Se presenta en lesiones de las extremidades inferiores de alta energía, entre los elementos clave para el diagnóstico se presenta dolor desmedido (Bango et al., 2020).

Los síntomas que caracterizan al síndrome compartimental agudo incluyen dolor, palidez, poiquilotermia, falta de pulso, parestesia y parálisis; las sospechas se confirman por medio de la medición de la presión intracompartimental del compartimento afectado, el tratamiento a utilizar es la fasciotomía oportuna (Pechar \& Lyons, 2016).

\section{PROCESOS INFECCIOSOS}

La curación ósea implica un proceso difícil de regeneración tisular, el cual está influenciado diferentes procesos biológicos y biomecánicos; en algunos casos es posible que estos procesos se dificulten debido a que no se han unido las partes, en otros casos la presencia de procesos infecciosos relacionados con la fractura (Foster et al., 2021). 
Una complicación de interés después de cualquier tratamiento quirúrgico de fracturas es la infección relacionada con la fractura (Hellebrekers et al., 2019). Los procesos infecciosos asociados con la osteosíntesis puede ser una complicación que desafíe la fijación de la fractura, agobiando de esta manera al paciente y a los cirujanos ortopédicos, el objetivo perseguido por los actuales tratamientos consiste en erradicar las infecciones, la curación de la fractura y la preservación de la función (Wong et al., 2020).

Con la finalidad de prevenir las infecciones en el sitio quirúrgico después de operaciones, es necesario realizar una evaluación del paciente y sus condiciones clínicas, disminuir la duración de la estadía del paciente en el centro de atención hospitalario, al igual que prevenir la presencia de complicaciones derivadas de procesos infecciosos (Pereira et al., 2015).

\section{DAÑO TENDINOSO-MUSCULAR}

Las fracturas que se producen como consecuencia de traumatismos severos tienen que pasar por un proceso fisiológico de recuperación, este puede tener algunas complicaciones, una de estas es el daño tendinoso-muscular (R. García et al., 2014). Los daños producidos en el mecanismo extensor ocasionan pérdidas significativas de la función, estas son frecuentes después de un traumatismo (Mahoney et al., 2021).

Algunas especies animales como los caninos y felinos domésticos sufren de daños en sus músculos y tendones como consecuencia de traumatismos, entre estos el músculo flexor digital superficial y su tendón, el tendón gastrocnemio y el tendón combinado de los músculos gracilis, semitendinoso y bíceps femoral, entre otros (American College of Veterinary Surgeons, 2021).

\section{ELEMENTOS DE OSTEOSÍNTESIS UTILIZADOS FRECUENTEMENTE EN EL TRATAMIENTO DE FRACTURAS APENDICULARES}

El procedimiento de elección para tratar huesos inestables y desplazados por fracturas es la osteosíntesis; algunas de las características deseables de los materiales utilizados para los implantes son: mayor biocompatibilidad, deben tener reabsorbibilidad predecible, suficiente resistencia mecánica para mantener estable la fractura y la degradación homogénea al tiempo que favorece la curación de la fractura (Holweg et al., 2020).

\section{TRATAMIENTO CERRADO U ORTOPÉDICO DE FRACTURAS APENDICULARES}

El propósito inicial de todo tratamiento en individuos que han sufrido fracturas está orientado en que este sea lo menos invasivo posible, en este caso la primera opción es la reducción cerrada, recuperar la alineación ósea y la estabilización mediante aparatos de tracción o compresión externa, sean estos 
valvas o yesos, la intención de estos es que la recuperación sea precoz por medio de la formación del callo externo estimulándolo con dinamismo muscular, movimiento articular y transferencia de carga (Rombolá, 2017a).

Mediante el uso de tratamientos cerrados es posible alcanzar la estabilidad mecánica generalmente entre 6 y 18 semanas, es decir, la recuperación es más lenta en lo que tiene que ver con la liberación de movilidad y carga axial; los métodos más utilizados de tratamientos cerrados de fracturas son los yesos de todo tipo y los aparatos de tracción, el primero de estos se utiliza para mantener las fracturas reducidas en posición, mientras que la tracción ya no se utiliza para el tratamiento de fracturas apendiculares, ha sido reemplazada por la fijación externa o interna (C. García \& Ortega, 2005).

\section{ELEMENTOS DE FIJACIÓN EXTERNA}

Los fijadores externos se han utilizado desde la época de Hipócrates, sin embargo, fue después de la Segunda Guerra Mundial cuando se hicieron grandes avances; el moderno fijador externo se produjo en la década de 1970, durante las últimas décadas se han producido avances significativos en la fijación externa, teniendo como principal característica la estabilidad (Sternick et al., 2012).

Los elementos de fijación externa se indican principalmente para la fijación temporal de fracturas producidas después de un traumatismo, también para corregir deformidades, tratamiento de defectos, entre otros casos en los que la intención es tener un daño mínimo de los tejidos blandos (Bliven et al., 2019). La fijación externa tiene algunas ventajas, entre estas: su aplicación es fácil, se puede obtener una buena reducción y estabilidad, su utilización involucra un bajo índice de complicaciones, entre otras (Álvarez \& García, 2017).

Entre las limitaciones de la fijación externa se incluyen el nivel significativo de habilidad y el entrenamiento necesarios para colocar alfileres y crear una estructura estable, es difícil de emplear en las articulaciones, es necesario que el paciente tenga bastante cumplimiento, el paciente necesita restringir su carga de peso sobre la extremidad afectada, debe mantenerse la asepsia, caso contrario se pueden presentarse procesos infecciosos (Cohn et al., 2017).

Los fijadores externos pueden ser alambres o agujas, los cuales se colocan en el hueso por encima y debajo del foco de fractura, se unen entre sí por barras verticales. De estos existen tres tipos: el estándar uniplanar, fijadores híbridos, es decir se combinan dos uniplanares o un uniplanar y un anular pegados al hueso con púas transfixiantes, estos dependen de los modelos que estén disponibles en el mercado, y el fijador anular, del cual uno de los más conocidos es el Ilizarov, esta forma una especie de exoesqueleto que circunda al hueso; se compone de marcos circulares fijos con alambres unidos entre sí por barras verticales (García \& Ortega, 2005). 


\section{ELEMENTOS DE FIJACIÓN INTERNA}

Una opción popular para tratar quirúrgicamente las fracturas apendiculares es la reducción artroscópica-fijación interna, aunque se han descrito varias técnicas, sigue siendo un desafío la reducción anatómica y la fijación estable de la articulación (Wolfson et al., 2021). Existen diferentes fijadores externos, cada uno de ellos posee diferente capacidad biomecánica, sin embargo, hasta la fecha no existe una inferencia acerca del método más eficaz a ser utilizado (Freitas et al., 2021).

\begin{tabular}{ll}
\hline \multicolumn{1}{c}{ Elemento } & \multicolumn{1}{c}{ Función } \\
\hline Golillas & $\begin{array}{l}\text {-Incrementan la superficie del área sobre el tornillo de fijación, previniendo la rotura } \\
\text { de la cortical } \\
\text {-Fijación de tendones avulsionados }\end{array}$ \\
\hline Grapas & -Fijación de artrodesis y osteotomías correctoras \\
\hline Agujas & -Guías para la introducción de tornillos \\
& -Tracción y fijación externa/interna transitoria o permanente \\
\hline Alambres & -Fijación para cerclajes y bandas de tensión \\
& -Cerclajes y bandas de tensión \\
\hline \multirow{2}{*}{ Tornillos } & -Fijación de placas al hueso-Compresión de dos fragmentos entre sí \\
& -Sujeción de ligamentos, tendones o cápsulas \\
\hline Placas & -De cortical o esponjosa, canulados con rosca y/o hilo \\
\hline Clavos & Compresión dinámica, neutralización y sostén \\
intramedulares & $\begin{array}{l}\text {-Inmovilización en fracturas con minutas muy inestables y con gran compromiso de } \\
\text { tejidos blandos } \\
\text {-Consolidación por formación de callo perióstico }\end{array}$ \\
\hline
\end{tabular}

(Rombolá, 2017)

La fijación interna submuscular, debido a sus propiedades biomecánicas, al igual que otras ventajas en su uso clínico, comodidad para el paciente, es una alternativa interesante a la fijación externa.

Golillas y grapas: las golillas tienen similitud con una arandela, su función consiste en distribuir la fuerza que puedan ejercer los tornillos sobre el hueso en áreas mayores, pudiendo evitar así una posible rotura (Rombolá, 2017).

Las grapas presentan forma de alambre, sus extremos terminan en punta y son utilizadas con la finalidad de unir dos elementos óseos que generalmente se sitúan en la zona articular de un hueso. Estas se pueden prefabricar o premoldear al momento de su utilización (Smith, 2010).

Agujas: estas pueden servir de utilidad para estabilizar fracturas como las radiales mediante osteosíntesis percutánea, se colocan de manera oblicua. Las agujas son parte esencial de algunos tratamientos quirúrgicos como el enclavado centromedular elástico estable (ECMEE); en fracturas diafisarias se las introduce en la cavidad medular tras trepanación de la cortical con una punta cuadrada para ascender hasta el foco de fractura (Fitoussi et al., 2020). 


\section{ALAMBRES}

El alambre de hierro se informa como material para la reparación de fracturas en 1775, en un manuscrito francés, la fijación más frecuente consistía en el uso de alambres de cerclaje para reparar fracturas. Joseph Lister, el padre de la asepsia, también utilizó alambres de metal para la reparación de fracturas abiertas y cerradas (Hernigou \& Pariat, 2016).

\section{ALAMBRES DE KIRSCHNER}

En los casos donde el uso de tornillos no es factible, el cirujano puede recurrir a una alternativa segura y confiable como son los alambres de Kirschner, estos pueden ser de utilidad en el caso de los injertos óseos vascularizados (Engel et al., 2020).

Tornillos: entre los métodos más utilizados para la fijación articular se encuentra el tornillo compresivo, el cual se ha demostrado que logra una compresión y estabilidad adecuada, además de buenos resultados clínicos y radiológicos. Su tasa de retiro es menor, así mismo, la tasa de seudoartrosis es más baja; entre sus desventajas se encuentran algunas limitantes para conseguir el ángulo de fijación deseado (Bennice et al., 2020).

Otro de los tornillos utilizados en el tratamiento de huesos largos es el tornillo interferencial tibial, este tiene algunas desventajas, puede empujar la plastia dentro del túnel, produciendo una pérdida de tensión, además, la escasa resistencia del hueso medular de la tibia proximal en ocasiones puede provocar una fijación interferencial inadecuada (L. García et al., 2020).

Placas: la reducción abierta y fijación interna mediante placas ofrece estabilidad apropiada para una movilización temprana y más intensa, sin embargo, algunas investigaciones han arrojado pobres resultados finales y alto número de complicaciones, esto ha hecho que, en la actualidad, su eficacia sea discutida (Gardenal et al., 2018).

Clavos intramedulares: debido a algunas complicaciones relacionadas con el uso de tornillos en el tratamiento de fracturas apendiculares, en la actualidad es recomendable realizar la síntesis de estos patrones inestables de fractura mediante el uso de clavos intramedulares. Estos dispositivos garantizan un entorno biomecánico superior al de los dispositivos extramedulares, además, reducen el riesgo de complicaciones (Jiménez et al., 2020).

Entre las ventajas del uso de clavos intramedulares se encuentran: menor agresión de partes blandas, menor tiempo quirúrgico, mínimo abordaje quirúrgico y su extracción es fácil (Jordà et al., 2020). La inflexibilidad del fijador, al igual que su resistencia a las cargas de compresión, flexión y rotación asociados al soporte del peso, pueden verse afectados por las características del clavo, el material de la 
barra conectora y la conformación del marco. El diámetro del clavo no debe exceder el 25\% del diámetro óseo (Cruz \& Gaviria, 2016).

\section{DISCUSIÓN}

A lo largo de esta investigación bibliográfica se destacan las principales complicaciones asociadas con las fracturas apendiculares en caninos, es importantes conocer estas para el momento de tratar un paciente con politraumatismos que comprometan el esqueleto apendicular, además de los diferentes elementos de osteosíntesis utilizados en este tipo de tratamiento de fracturas.

Ante la presencia de un paciente politraumatizado con fractura del esqueleto apendicular, se suelen lesionar otros tejidos aparte del hueso, por esta razón, los cuidados iniciales que se dé al paciente deben estar encaminados hacia el daño sistémico sufrido por el paciente, además de las consecuencias a nivel sistémico que pueda ocasionar el daño musculoesquelético.

Un tema importante en el trabajo del médico veterinario ha sido el abordaje radiológico de los pacientes politraumatizados, esto se debe al arsenal de elementos de fijación utilizados para tratar al paciente, por lo cual, en esta investigación se ha realizado un análisis y descripción de cada uno de los mecanismos de fijación utilizados, características, principios biomecánicos y posibles complicaciones.

En la actualidad los métodos a los que se recurre con mayor para el tratamiento de fracturas apendiculares son los métodos de fijación interna, principalmente los tornillos, placas y clavos intramedulares, de estos. Autores como Jiménez, 2020, recomiendan el uso de clavos intramedulares para el tratamiento de fracturas apendiculares debido a algunas complicaciones relacionadas con el uso de tornillos, además, debido a sus ventajas, entre estas: menor agresión de partes blandas, menor tiempo quirúrgico, mínimo abordaje quirúrgico y su extracción es fácil. 


\section{REFERENCIAS BIBLIOGRÁFICAS}

Agbelele, P., Van Maris, F., Sanguina, M., Zerkly, B., Djebara, A. E., \& Girard, P. (2020). Use of chest CT-scan images to differentiate between SARS-CoV-2 infection and fat embolism: A clinical case. Radiology Case Reports, 15(10), 2047-2050. https://doi.org/10.1016/j.radcr.2020.07.071

Almeida, L., Pereira, T., \& de Carvalho, F. (2020). Propostas, recursos e resultados nas ciências da saúde 2. In Atena Editora (Primera ed, Issue December). https://doi.org/10.22533/at.ed.35020240612

Álvarez, A., \& García, Y. (2017). Fijación externa en la fracturas distal del radio. Revista Archivo Médico de Camagüey, 21(4), 546-556. http://scielo.sld.cu/scielo.php?script=sci_arttext\&pid=S102502552017000400014

American College of Veterinary Surgeons. (2021). Lesiones del Tendón de Aquiles. Lesiones Del Tendón de Aquiles. https://www.acvs.org/small-animal-es/achilles-tendon-injuries

Balakrishnan, A. (2020). Resuscitation Strategies for the Small Animal Trauma Patient. Veterinary Clinics of North America - Small Animal Practice, 50(6), 1385-1396. https://doi.org/10.1016/j.cvsm.2020.07.012

Bango, J., Zhang, E., Aaron, D., \& Diwan, A. (2020). Two cases of acute anterolateral compartment syndrome following inversion ankle injuries. Trauma Case Reports, 30(November), 100371. https://doi.org/10.1016/j.tcr.2020.100371

Bennice, J., Gallucci, G. L., Zaidenberg, E. E., De Carli, P., \& Boretto, J. G. (2020). Estudio comparativo entre banda de tensión y tornillo compresivo para la artrodesis interfalángica proximal y metacarpofalángica. Revista de La Asociación Argentina de Ortopedia y Traumatología, 85(4), 325-334. https://doi.org/10.15417/issn.1852-7434.2020.85.4.1063

Bliven, E., Greinwald, M., Hackl, S., \& Augat, P. (2019). External fixation of the lower extremities: Biomechanical perspective and recent innovations. Injury, 50(1), S10-S17. https://doi.org/10.1016/j.injury.2019.03.041

Burr, T., Chaudhry, H., Zhang, C., Vasilopoulos, V., \& Allam, E. (2020). Fat embolism in the popliteal vein detected on CT: Case report and review of the literature. Radiology Case Reports, 15(11), 23082313. https://doi.org/10.1016/j.radcr.2020.09.008

Butcher, N., D’Este, C., \& Balogh, Z. (2014). The quest for a universal definition of polytrauma: A trauma registry-based validation study. Journal of Trauma and Acute Care Surgery, 77(4), 620-623. https://doi.org/10.1097/TA.0000000000000404

Byers, C. (2017). Fluid Therapy: Options and Rational Selection. Veterinary Clinics of North America Small Animal Practice, 47(2), 359-371. https://doi.org/10.1016/j.cvsm.2016.09.007

Cohn, M., Unnanuntana, A., Pannu, T., Warner, S., \& Lane, J. (2017). Materials in fracture fixation. In Comprehensive Biomaterials II (Primera ed, Vol. 7, Issue September 2016, pp. 278-297). https://doi.org/10.1016/B978-0-12-803581-8.10109-2

Cruz, J. M., \& Gaviria, A. (2016). El fijador esquelético externo: aplicación clínica en perros y gatos. Revista de Medicina Veterinaria, 1(32), 109. https://doi.org/10.19052/mv.3860 
De Morais, L., Portolan, B., \& Mendonça, D. (2015). Caracterização de lesões e alterações clínicas de pacientes politraumatizados atendidos no Hospital Veterinário Da Uniju. XXIII Seminário de Iniciação Científica, 5-8. https://cutt.ly/ix2vLtH

Engel, H., Xiong, L., Heffinger, C., Kneser, U., \& Hirche, C. (2020). Comparative outcome analysis of internal screw fixation and Kirschner wire fixation in the treatment of scaphoid nonunion. Journal of Plastic, Reconstructive and Aesthetic Surgery, 73(9), 1675-1682. https://doi.org/10.1016/j.bjps.2020.03.016

Fitoussi, F., Alves, A., \& Bachy, M. (2020). Fracturas diafisarias del antebrazo en los niños. EMC Aparato Locomotor, 53(1), 1-12. https://doi.org/10.1016/s1286-935x(20)43375-1

Foster, A., Moriarty, I., Zalavras, C., Morgenstern, M., Jaiprakash, A., Crawford, R., Burch, M. A., Boot, W., Tetsworth, K., Miclau, T., Ochsner, P., Schuetz, M., Richards, G., \& Metsemakers, W. J. (2021). The influence of biomechanical stability on bone healing and fracture-related infection: the legacy of Stephan Perren. Injury, 52(1), 43-52. https://doi.org/10.1016/j.injury.2020.06.044

Freitas, A., Toledo, J., Ferreira, A., Aquino, R., Leão, V., \& Péricles, W. (2021). Biomechanical study of different internal fixations in Pauwels type III femoral neck fracture - A finite elements analysis. Journal of Clinical Orthopaedics and Trauma, 14(xxxx), 145-150. https://doi.org/10.1016/j.jcot.2020.06.006

García, C., \& Ortega, D. (2005). Elementos de osteosíntesis de uso habitual en fraturas del esqueleto apendicular: evaluación radiológica. Revista Chilena de Radiología, 11(2), 58-70. https://scielo.conicyt.cl/pdf/rchradiol/v11n2/art05.pdf

García, L., Ortega, A., García, M., Castelló, A., \& Sertafy, J. (2020). Fracaso de la reconstrucción del ligamento cruzado anterior. Revisión de la literatura. Revista Española de Traumatología Laboral, 3(1), 37-52. https://doi.org/https://doi.org/10.24129/j.retla.03105.fs2005007

García, R., Benhamú, S., Cortés, E., \& Gijón, G. (2014). Síndrome compartimental y síndrome de Sudeck secundario a fractura de talón: compensaciones ortopédicas en un caso clínico. Revista Española de Podología, 25(3), 104-109. https://cutt.ly/9vofNeW

Gardenal, R. M., Seri, M. S., Faccendini, S. J., Kalejman, G. B., \& Bichara, J. A. (2018). Fracturas de falange tratadas con placas de osteosíntesis. Resultados a corto plazo. Revista de La Asociación Argentina de Ortopedia y Traumatología, 83(3), 179. https://doi.org/10.15417/issn.1852-7434.2018.83.3.661

Hellebrekers, P., Rentenaar, R. J., McNally, M. A., Hietbrink, F., Houwert, R. M., Leenen, L. P. H., \& Govaert, G. A. M. (2019). Getting it right first time: The importance of a structured tissue sampling protocol for diagnosing fracture-related infections. Injury, 50(10), 1649-1655. https://doi.org/10.1016/j.injury.2019.05.014

Hernigou, P., \& Pariat, J. (2016). History of internal fixation ( part 1 ): early developments with wires and plates before World War II. International Orthopaedics, 41(part 1), 1273-1283. https://doi.org/10.1007/s00264-016-3347-4

Hitosugi, M., Takeda, A., Hiraizumi, S., \& Moriguchi, S. (2019). Liver tissue embolism in the portal vein. Romanian Journal of Legal Medicine, 27(2), 119-121. https://doi.org/10.4323/rjlm.2019.119 
Holweg, P., Berger, L., Cihova, M., Donohue, N., Clement, B., Schwarze, U., Sommer, N. G., Hohenberger, G., van den Beucken, J., Seibert, F., Leithner, A., Löffler, J., \& Weinberg, A. (2020). A lean magnesium-zinc-calcium alloy ZX00 used for bone fracture stabilization in a large growing-animal model. Acta Biomaterialia, 113(1), 646-659. https://doi.org/10.1016/j.actbio.2020.06.013

Hsieh, C. H., Chen, Y. C., Hsu, S. Y., Hsieh, H. Y., \& Chien, P. C. (2018). Defining polytrauma by abbreviated injury scale $\geq 3$ for a least two body regions is insufficient in terms of short-term outcome: A cross-sectional study at a level I trauma center. Biomedical Journal, 41(5), 321-327. https://doi.org/10.1016/j.bj.2018.08.007

Jiménez, V., Auñón, I., Pardo, J., Olaya, C., \& Caba, P. (2020). ¿Afecta la fractura de la pared lateral al grado de colapso y al grado de deslizamiento de la lámina cefálica, en fracturas pertrocantéreas tratadas mediante enclavado intramedular? Estudio radiológico y revisión de la literatura. Revista Espanola de Cirugia Ortopedica y Traumatologia, 65(2), 108-115. https://doi.org/10.1016/j.recot.2020.06.013

Jordà, P., Antequera, J., Ferràs, J., Blasco, M., Mascarell, A., Puchol, E., \& Salom, M. (2020). Buried intramedullary implants for paediatric forearm fractures. Does the refracture rate improve? Revista Española de Cirugia Ortopedica y Traumatologia, 64(1), 35-40. https://doi.org/10.1016/j.recot.2019.07.004

Llewellyn, E., Lourenço, M., \& Ambury, A. (2020). Recognition, treatment, and monitoring of canine hypovolemic shock in first opinion practice in the United Kingdom. Topics in Companion Animal Medicine, 39(1), 1-39. https://doi.org/10.1016/j.tcam.2020.100427

Mahmoud, O., \& Mahmoud, M. (2018). Spectral Doppler findings in a rare case of acute compartment syndrome following leg burn. Radiology Case Reports, 13(2), 352-355. https://doi.org/10.1016/j.radcr.2018.01.006

Mahoney, R., Veravalli, K., \& Mofidi, A. (2021). Treatment of neglected quadriceps tendon ruptures using LARS ${ }^{\mathrm{TM}}$ ligament augmentation: A case series. Trauma Case Reports, 32(1), 100437. https://doi.org/10.1016/j.tcr.2021.100437

Pechar, J., \& Lyons, M. (2016). Acute Compartment Syndrome of the Lower Leg: A Review. Journal for Nurse Practitioners, 12(4), 265-270. https://doi.org/10.1016/j.nurpra.2015.10.013

Pereira, H., Rezende, E., \& Marinho, B. (2015). Length of preoperative hospital stay: a risk factor for reducing surgical infection in femoral fracture cases. Revista Brasileira de Ortopedia, 50(6), 638-646. https://doi.org/10.1016/j.rboe.2015.09.006

Rombolá, E. (2017a). Evaluación radiológica de los elementos de osteosíntesis en el miembro superior. Revista Argentina de Radiologia, 81(4), 285-295. https://doi.org/10.1016/j.rard.2016.11.007

Rombolá, E. (2017b). Evaluación radiológica de los elementos de osteosíntesis en el miembro superior. Revista Argentina de Radiología, 81(4), 285-295. https://doi.org/10.1016/j.rard.2016.11.007

Rothberg, D., \& Makarewich, C. (2018). Fat Embolism and Fat Embolism Syndrome. Journal of the American Academy of Orthopaedic Surgeons, 00(00), 1-10. https://doi.org/10.5435/JAAOS-D-17-00571

Rozanski, E., \& Rondeau, M. (2002). Choosing fluids in traumatic hypovolemic shock: The role of crystalloids, colloids, and hypertonic saline. Journal of the American Animal Hospital Association, 38(6), 
499-501. https://doi.org/10.5326/0380499

Sánchez, G., Nasello, W., \& Sappia, D. (2017). Complicaciones asociadas a fracturas apendiculares en caninos politraumatizados [Universidad Nacional del Centro de la Provincia de Buenos Aires]. https://cutt.ly/0x2HW2M

Smith, T. F. (2010). Bone Staple: Tried and True Superhero of Bone Fixation. In Bone Staple (pp. 240245). http://www.podiatryinstitute.com/pdfs/Update_2010/2010_41.pdf

Sohn, H. S., Chung, J. Y., \& Song, H. K. (2019). Analysis of complications and clinical outcomes in the treatment of segmental tibial fractures according to the method of internal fixation. Asian Journal of Surgery, 42(7), 740-745. https://doi.org/10.1016/j.asjsur.2018.11.001

Sternick, M., Dallacosta, D., Bento, D. Á., \& do Reis, M. (2012). Relationship Between Rigidity of External Fixator and Number of Pins: Computer Analysis Using Finite Elements. Revista Brasileira de Ortopedia (English Edition), 47(5), 646-650. https://doi.org/10.1016/s2255-4971(15)30017-3

von Ritgen, S., Böhler, A., Rosser, J., Haralambus, R., \& Florczyk, A. (2017). Polytrauma Patient With Upper Airway Obstruction-Case Report. Journal of Equine Veterinary Science, 51, 110-112. https://doi.org/10.1016/j.jevs.2016.11.012

Wolfson, T., Vadhera, A. S., Parvaresh, K., Verma, N., LaPrade, R., \& Chahla, J. (2021). Arthroscopic Reduction and Internal Fixation of Tibial Eminence Fractures With Transosseous Suture Bridge Fixation. Arthroscopy Techniques, 10(4), e1039-e1046. https://doi.org/10.1016/j.eats.2020.12.003

Wong, R., Li, T. kiu, Li, J., Ho, W., Chow, S., Leung, S., Cheung, W., \& Ip, M. (2020). A systematic review on current osteosynthesis-associated infection animal fracture models. Journal of Orthopaedic Translation, 23(February), 8-20. https://doi.org/10.1016/j.jot.2020.03.002 